\title{
Carga imediata - da exodontia à restauração cerâmica em 30 dias: relato de caso
}

Immediate load - the exodontia to ceramic restoration in 30 days: case report

GONÇALVES JÚNIOR, Ubiratan'; FERNANDES, Samuel Lucas'; ${ }^{2}$ SOUSA, Matheus Ferreira Silva ${ }^{3}$; BOER, Nagib Pezati ${ }^{4}$;ÓBREGA, Adhara Smith ${ }^{5}$; ARRUDA, Farid Jamil Silva ${ }^{6}$

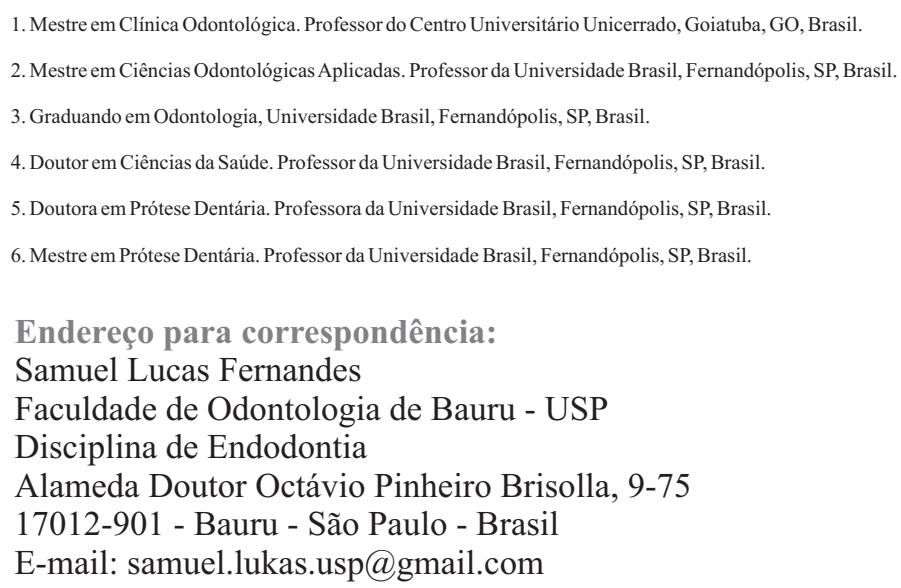

\section{RESUMO}

O reestabelecimento estético e funcional é um dos principais objetivos do paciente quando procura o cirurgião dentista. Os implantes permitem que os objetivos do tratamento sejam atingidos de forma satisfatória, sendo que osseointegração bemsucedida irá garantir a estabilidade dos implantes para suportar a prótese e a carga mastigatória ao longo dos anos. Atualmente tem sido lançado no mercado os implantes Unitite, SIN (Sistema de Implante, São Paulo, SP, Brasil), com novos conceitos de design e tratamento de superfície, tem como objetivos acelerar a cicatrização e a osseointegração. O objetivo deste artigo é relatar um caso clínico onde da exodontia à restauração cerâmica foram gastos 30 dias.

Palavras-chave: Implantes dentários. Osseointegração. Cirurgia bucal.

\footnotetext{
ABSTRACT

The aesthetic and functional reestablishment is a key objective of the patient when looking for the dentist. The implants allow the treatment goals to satisfactorily achieved, and successful osseointegration will ensure the stability of the implants to support the prosthesis and the masticatory load over the years. Currently Unitite implants, SIN (Implant System, São Paulo, SP, Brazil), with new design and surface treatment concepts, have been launched on the market, aiming to accelerate healing and osseointegration. The objective of this article is to report a clinical case where from the exodontia to the ceramic restoration in 30 days.
}

Keywords: Dental implants. Osseointegration. Surgery, oral. 


\section{INTRODUÇÃO}

A obtenção de estabilidade primária após a colocação do implante foi considerada um dos fatores essenciais para a realização da estabilidade secundária (osseointegração) ${ }^{1}$. Níveis de estabilidade primária insuficientes resultam em micro movimentos, e uma magnitude acima de 50-150 ?m normalmente resulta em encapsulamento do implante por tecido mole ${ }^{2}$.

Para se obter sucesso na osseointegração e na estabilidade primária, distintas estratégias têm sido desenvolvidas, sendo principalmente relacionadas à superfície $^{3}$ e à geometria ${ }^{4}$ do implante. Além disso o sistema de preparação do sítio cirúrgico mostrou influenciar essa estabilidade1. Existe ainda a hipótese de que o tratamento de superfície poderá melhorar a osseointegração promovendo maior aposição óssea, o que levaria a um aumento no contato osso-implante ${ }^{3}$.

Recentemente o sistema Unitite da empresa SIN foi lançado no mercado com a proposta de acelerar o processo de osseointegração. Este implante possui macrogeometria híbrida com câmaras de cicatrização e tratamento de superfície com duplo ataque ácido ativado com nanocristais de hidroxiapatita (Unitite com Superfície SINactive, SIN Sistema de Implante, São Paulo, SP, Brasil) $)^{5}$.

$\mathrm{O}$ objetivo do presente artigo é relatar através de um caso clínico a colocação de um implante unitário na região de incisivo lateral superior com carga imediata, em que da exodontia à restauração cerâmica foram gastos 30 dias.

\section{RELATO DE CASO}

Um paciente leucoderma, 56 anos, apresentou-se a clínica particular com a indicação da exodontia do elemento 12 , ao exame radiográfico constatou-se de uma fratura radicular no referido dente (Figura 1). O paciente apresentava uma boa condição de saúde geral e o planejamento pautou-se na exodontia e colocação imediata de implante com provisório.

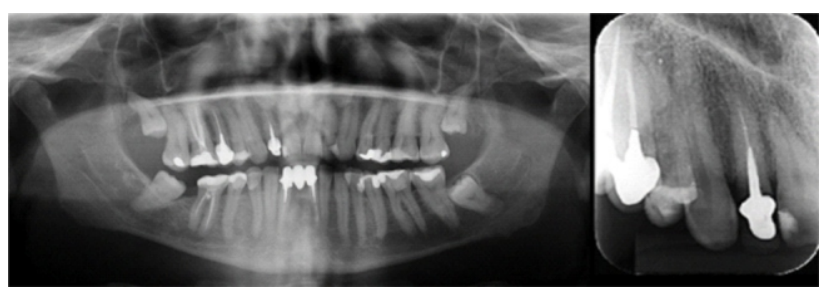

Figura 1 - Radiografias panorâmica e periapical iniciais.
Um Implante SIN Unitite Slin de plataforma $2.9 \mathrm{~mm}$ de diâmetro por $11.5 \mathrm{~mm}$ de comprimento, foi instalado após a exodontia e colocado uma prótese provisória na mesma sessão. O implante foi posicionado para realizar prótese cimentada, instalando um abutment universal reto e confeccionado provisório sobre o mesmo.

Após 30 dias foi realizada a moldagem para a confecção da coroa de porcelana. Que foi instalada um dia depois da moldagem.

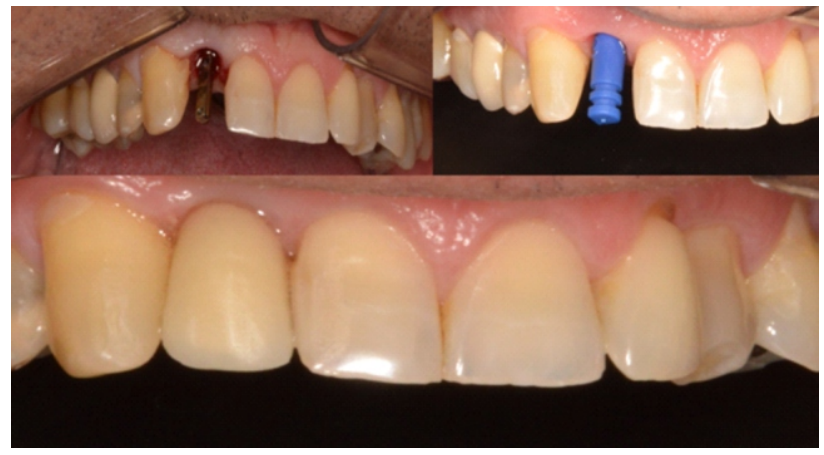

Figura 2 - Instalação do provisório imediato.

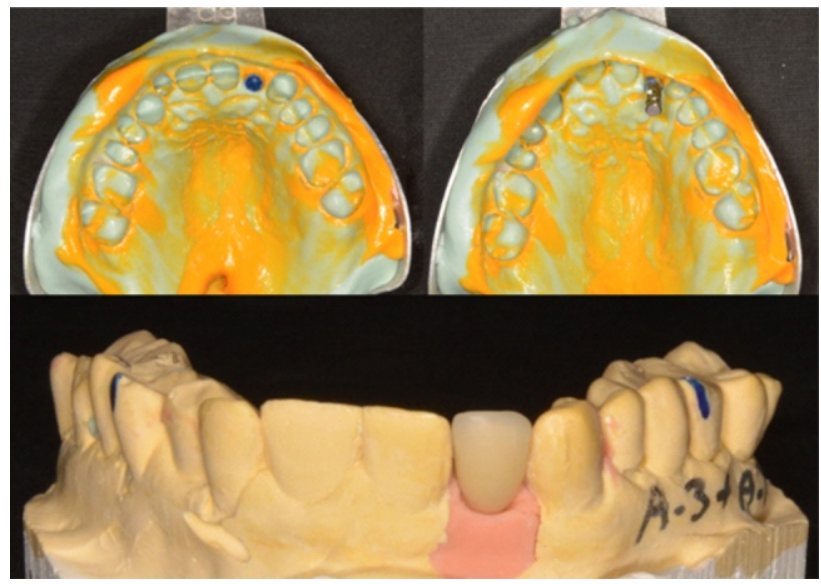

Figura 3 - Moldagem de transferência e coroa de porcelana adaptada no modelo.

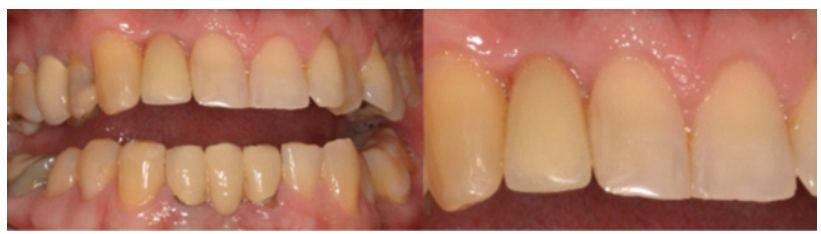

Figura 4 - Aspecto final após a cimentação da porcelana.

\section{DISCUSSÃO}

O processo que envolve a remoção de um dente, e a instalação de implantes com posterior reabilitação protética, quando feito em diversas etapas separadas por 
longos períodos geram expectativa e consequente ansiedade no paciente à espera do resultado. Atender a expectativas do paciente é de fundamental importância para o sucesso do tratamento ${ }^{6}$, apesar disso é importante se atentar para os principais objetivos da reabilitação com implantes que é um ótimo resultado de tratamento estético com alta previsibilidade e baixo risco de complicação? .

Prevenir a reabsorção óssea alveolar com um procedimento conservador no momento da extração pode aumentar a estética e reduzir a duração e extensão do tratamento necessário para a colocação do implante tratamento ${ }^{6}$.

A remoção do dente e colocação do implante imediatamente após a remoção, possibilita acelerar a reabilitação ${ }^{8}$, esse conceito ganha cava vez mais espaço por ser um procedimento rápido, demandando menos tempo ao profissional e ao paciente, melhorando assim a aceitação dos mesmos ${ }^{9}$. Todavia o paciente ainda pode aguardar um longo tempo, quando não há condições clínicas ideais para a colocação de uma prótese imediata sobre o implante instalado $^{10}$. Atualmente se busca alternativas para acelerar esse processo. E alterações que favoreçam um melhor travamento inicial e posterior aceleração no processo de cicatrização e osseointegração são propostos.

Neste caso optou-se por um sistema de implantes que reúne diversas características visando atender as expectativas e o planejamento realizado. O implante escolhido foi o Unitite Slim que apresenta diversas possibilidades, com três medidas distintas de comprimento e apenas $2,9 \mathrm{~mm}$ de diâmetro. Esse sistema de implante foi desenvolvido para dar mais conforto as reabilitações em áreas estreitas e espaços interdentais limitados, assim como regiões de incisivos laterais superiores e incisivos inferiores. Suas dimensões reduzidas evita acidentes e possibilita uma boa vascularização, facilitando o processo de osseointegração.

O sistema de conexão tipo Cone Morse apresenta uma superioridade biomecânica dos acoplamentos protéticos com ângulo interno de 3 graus. E tem sua eficácia comprovada em reabilitações de regiões estéticas, proporcionando uma boa condição gengival e uma perda óssea ínfima.

A colocação da carga imediata foi considerada pois o caso atendia a condições essenciais para a sua indicação, sendo o sucesso creditado ao fato de se obedecer essas condições, que segundo a literatura vigente se atendidas irão levar ao sucesso clínico na esmagadora maioria dos $\operatorname{casos}^{9,11}$.

Os resultados foram alcançados e em trinta dias o paciente já estava com o tratamento concluído, restando apenas a proservação do caso. $\mathrm{O}$ que se observa analisando deste caso é que os implantes com essa proposta estão prontos para revolucionar mais uma vez os conceitos de reabilitação utilizando-se de implantes osseointegrados, proporcionando reabilitações rápidas e definitivas com segurança.

\section{CONCLUSÃO}

O sistema de implantes SIN Unitite Slin proporcionou o restabelecimento estético e funcional do paciente, deixando-o extremamente satisfeito com o resultado do caso e com a velocidade da resolução do mesmo.

\section{REFERÊNCIAS}

1. Campos FE, Jimbo R, Bonfante EA, Barbosa DZ, Oliveira MT, Janal $\mathrm{MN}$, et al. Are insertion torque and early osseointegration proportional? A histologic evaluation. Clin Oral Implants Res. 2015;26(11):1256-60.

2. Szmukler-Moncler S, Salama H, Reingewirtz Y, Dubruille JH. Timing of loading and effect of micromotion on bone-dental implant interface: review of experimental literature. J Biomed Mater Res. 1998;43(2):192-203.

3. Buser D, Broggini N, Wieland M, Schenk RK, Denzer AJ, Cochran DL, et al. Enhanced bone apposition to a chemically modified SLA titanium surface. J Dent Res. 2004;83(7):529-33.

4. Chowdhary R, Halldin A, Jimbo R, Wennerberg A. Influence of micro threads alteration on osseointegration and primary stability of implants: an FEA and in vivo analysis in rabbits. Clin Implant Dent Relat Res. 2015;17(3):562-9.

5. Bezerra FP, Pessoa RS, Zambuzzi WF. Carregamento funcional imediato ou precoce de implantes com câmara de cicatrização e nanosuperfície: estudo clínico prospectivo longitudinal. Innov Implant J Biomater Esthet. 2014;9(2/3):13-7.

6. Klokkevold PR, Han TJ, Camargo PM. Aesthetic management of extractions for implant site development: delayed versus staged implant placement. Pract Periodontics Aesthet Dent. 1999;11(5):603-10; quiz 612.

7. Buser D, Chen ST. Implant placement in postextraction sites. In: Buser D, editor. 20 years of guided bone regeneration in implant dentistry. 2nd ed. Chicago: Quintessence; 2009. p. 153-94.

8. Chen ST, Buser D. Esthetic outcomes following immediate and early implant placement in the anterior maxilla - a systematic review. Int J Oral Maxillofac Implants. 2014;Suppl:186-215.

9. Strub JR, Jurdzik BA, Tuna T. Prognosis of immediately loaded implants and their restorations: a systematic literature review. J Oral Rehab. 2012;39(9):704-17.

10. Youssef PI, Simões AX, Zielak JC, Giovanini AF, Deliberador TM, Campos EA. Carga imediata sobre implantes dentários. Rev Sul-Bras Odontol. 2009;6(4):441-6.

11. Xu L, Wang X, Zhang Q, Yang W, Zhu W, Zhao K. Immediate versus early loading of flapless placed dental implants: a systematic review. J Prosthet Dent. 2014;112(4):760-9. 